\title{
The Linear Fractional Model Theorem and Aleksandrov-Clark measures
}

\author{
Nieminen, Pekka J.
}

2015

Nieminen , P J \& Gallardo-Gutiérrez , E A 2015 , ' The Linear Fractional Model Theorem and Aleksandrov-Clark measures ' , Journal of the London Mathematical Society , vol. 91 , no. 2 , pp. 596-608 . https://doi.org/10.1112/jlms/jdv002

http://hdl.handle.net/10138/155184

https://doi.org/10.1112/jlms/jdv002

publishedVersion

Downloaded from Helda, University of Helsinki institutional repository.

This is an electronic reprint of the original article.

This reprint may differ from the original in pagination and typographic detail.

Please cite the original version. 


\title{
The Linear Fractional Model Theorem and Aleksandrov-Clark measures
}

\author{
Eva A. Gallardo-Gutiérrez and Pekka J. Nieminen
}

\begin{abstract}
A remarkable result by Denjoy and Wolff states that every analytic self-map $\varphi$ of the open unit disc $\mathbb{D}$ of the complex plane, except an elliptic automorphism, has an attractive fixed point to which the sequence of iterates $\left\{\varphi_{n}\right\}_{n \geqslant 1}$ converges uniformly on compact sets: if there is no fixed point in $\mathbb{D}$, then there is a unique boundary fixed point that does the job, called the DenjoyWolff point. This point provides a classification of the analytic self-maps of $\mathbb{D}$ into four types: maps with interior fixed point, hyperbolic maps, parabolic automorphism maps and parabolic non-automorphism maps. We determine the convergence of the Aleksandrov-Clark measures associated to maps falling in each group of such classification.
\end{abstract}

\section{Introduction}

Let $\mathbb{D}$ denote the open unit disc of the complex plane and $\varphi$ be an analytic map taking $\mathbb{D}$ into itself. If $\varphi$ has a fixed point $p$ in $\mathbb{D}$ and is not an automorphism, then an argument based on the Schwarz Lemma shows that the sequence of iterates

$$
\varphi_{n}=\varphi \circ \cdots \circ \varphi \quad(n \text { times })
$$

converges to $p$ uniformly on compact subsets of $\mathbb{D}$. The Denjoy-Wolff Theorem makes a striking assertion: If $\varphi$ has no fixed point in $\mathbb{D}$, then there is still a (necessarily unique) point $p$ in $\mathbb{T}$, the unit circle, such that $\left\{\varphi_{n}\right\}$ converges to $p$ uniformly on compact subsets of $\mathbb{D}$. This point, called the Denjoy-Wolff point of $\varphi$ behaves like a fixed point of $\varphi$, that is, $\varphi$ has radial (in fact non-tangential) limit $p$ at $p$ (see, for example, [16, Chapter 5]).

Moreover, it follows from the Julia-Carathéodory Theorem that the Denjoy-Wolff point is a 'point of conformality' in the sense of angular derivatives. More precisely, the non-tangential limit

$$
\varphi^{\prime}(p)=\angle \lim _{z \rightarrow p} \frac{\varphi(z)-p}{z-p},
$$

exists in $(0,1]$. Furthermore, a converse of the Denjoy-Wolff theorem also holds: If $p \in \mathbb{T}$ is any radial-limit fixed point of a non-identity function $\varphi$ and $0<\varphi^{\prime}(p) \leqslant 1$, then $\varphi$ has no fixed points in $\mathbb{D}$ and $\left\{\varphi_{n}\right\}$ converges to $p$ uniformly on compact subsets of $\mathbb{D}$, that is, $p$ is the Denjoy-Wolff point of $\varphi$. In such a case, the Julia-Carathéodory Theorem also asserts that

$$
\varphi^{\prime}(p)=\angle \lim _{z \rightarrow p} \varphi^{\prime}(z)=\liminf _{z \rightarrow p} \frac{1-|\varphi(z)|}{1-|z|},
$$

and $\varphi^{\prime}(p)$ is called the angular derivative of $\varphi$ at $p$.

Received 8 July 2014.

2010 Mathematics Subject Classification 30D05 (primary).

The first author was partially supported by Plan Nacional I+D grant no. MTM2013-42105-P. The second author was partially supported by the Finnish CoE in Analysis and Dynamics Research. 


\subsection{The Linear Fractional Model Theorem}

Motivated by the restrictions on the value of the angular derivative that an analytic selfmap of $\mathbb{D}$ can take at its Denjoy-Wolff point, it is possible to introduce the following general classification (see [2], Introduction).

Definition 1.1. An analytic self-map $\varphi$ of $\mathbb{D}$ is of

(1) elliptic type if it has a fixed point in $\mathbb{D}$;

(2) hyperbolic type if it has no fixed point in $\mathbb{D}$ and has angular derivative strictly less than 1 at its Denjoy-Wolff point;

(3) parabolic type if it has no fixed point in $\mathbb{D}$ and has angular derivative equal to 1 at its Denjoy-Wolff point.

With this classification at hand, the Linear Fractional Model Theorem asserts, in some sense, that every analytic self-map $\varphi$ of $\mathbb{D}$ is modelled by a linear fractional map. More precisely, let $p$ denote the fixed point or Denjoy-Wolff point of $\varphi$. If $\varphi$ is of elliptic type and $\varphi^{\prime}(p) \neq 0$, then Koenigs [8] proved in 1884 that there exists a non-trivial analytic mapping $\sigma$ on $\mathbb{D}$ such that Schröder's equation

$$
\sigma \circ \varphi=c \sigma
$$

holds with $c=\varphi^{\prime}(p)$. In 1931, Valiron [17] proved that if $\varphi$ is of hyperbolic type, then the same functional equation holds for $c=1 / \varphi^{\prime}(p)$ and an analytic mapping $\sigma$, taking $\mathbb{D}$ into the right half-plane and sending $p$ to $\infty$.

The parabolic case was addressed in 1979 by Pommerenke [12] and Baker and Pommerenke [1] , and independently by Cowen [5], who showed that it separates into two subcases, distinguished by the behaviour of orbits relative to the pseudo-hyperbolic metric in $\mathbb{D}$, denoted here by $\rho$. In particular, they proved that there exists an analytic mapping $\sigma$ on $\mathbb{D}$ such that either

$$
\sigma \circ \varphi=\sigma+i a
$$

for some real $a$, where the image of $\sigma$ can be taken to lie in the right half-plane, or

$$
\sigma \circ \varphi=\sigma+1 \text {. }
$$

The former case arises when the orbits of $\varphi$ are pseudo-hyperbolically separated in the sense that

$$
\inf _{n} \rho\left(\varphi_{n+1}(z), \varphi_{n}(z)\right)>0
$$

and the latter arises when they are not:

$$
\inf _{n} \rho\left(\varphi_{n+1}(z), \varphi_{n}(z)\right)=0 .
$$

It turns out that this separation dichotomy is independent of the base point: if it holds for one $z \in \mathbb{D}$, then it holds for all $z \in \mathbb{D}$. Thus the maps of parabolic type fall into two subclasses: positive hyperbolic step case (or automorphic type) if the orbits are separated, and zero hyperbolic step (or non-automorphic type) if they are not. The distinction between those subcases is the most subtle aspect of the Linear Fractional Model Theorem.

For a unified approach to all the cases of the Linear Fractional Model Theorem, we refer to Cowen's paper [5] and the book [6, Section 2.4].

\subsection{Aleksandrov-Clark measures}

We next collect some basic facts about Aleksandrov-Clark measures. For more information on these measures, we refer the reader to $[\mathbf{3}, \mathbf{9}, \mathbf{1 1}, \mathbf{1 4}]$. 
Let $\varphi$ be an analytic self-map of $\mathbb{D}$. For any $\alpha \in \mathbb{T}$, an easy computation shows that the real part of the function $(\alpha+\varphi) /(\alpha-\varphi)$ is positive and harmonic in $\mathbb{D}$, so it may be expressed as the Poisson integral of a positive Borel measure $\tau_{\alpha}$ supported on $\mathbb{T}$. That is,

$$
\operatorname{Re} \frac{\alpha+\varphi(z)}{\alpha-\varphi(z)}=\frac{1-|\varphi(z)|^{2}}{|\alpha-\varphi(z)|^{2}}=\int_{\mathbb{T}} P_{z} d \tau_{\alpha},
$$

where

$$
P_{z}(\zeta)=\frac{1-|z|^{2}}{|\zeta-z|^{2}}
$$

is the Poisson kernel for $z \in \mathbb{D}$. The family of measures $\left\{\tau_{\alpha}: \alpha \in \mathbb{T}\right\}$ are called the AleksandrovClark measures associated to $\varphi$. By means of the Herglotz formula, it holds that

$$
\frac{\alpha+\varphi(z)}{\alpha-\varphi(z)}=\int_{\mathbb{T}} \frac{\zeta+z}{\zeta-z} d \tau_{\alpha}(\zeta)+i c_{\alpha}
$$

where

$$
c_{\alpha}=\operatorname{Im} \frac{\alpha+\varphi(0)}{\alpha-\varphi(0)} .
$$

Note that if $\alpha$ is given and $\tau$ is any positive and finite Borel measure on $\mathbb{T}$, then formula (1.1) can be used to construct a map $\varphi$ whose Aleksandrov-Clark measure at $\alpha$ equals $\tau$.

For any Borel measure $\tau$ on $\mathbb{T}$, we write $d \tau=\tau^{a} d m+d \tau^{s}$ for the Lebesgue decomposition of $\tau$, where $\tau^{a}$ is the density of the absolutely continuous part, $m$ is the normalized Lebesgue measure on $\mathbb{T}$ and $\tau^{s}$ is singular. It follows from the basic properties of Poisson integrals that $\tau_{\alpha}^{s}$ is carried by the set where $\varphi(\zeta)=\alpha$ and

$$
\tau_{\alpha}^{a}(\zeta)=\frac{1-|\varphi(\zeta)|^{2}}{|\alpha-\varphi(\zeta)|^{2}}
$$

In particular, $\tau_{\alpha}$ is singular if and only if $\varphi$ is an inner function.

Let us recall that if the quotient $(\varphi(z)-\eta) /(z-\zeta)$ has a finite non-tangential limit at $\zeta \in \mathbb{T}$ for some $\eta \in \mathbb{T}$, then this limit is called the angular derivative of $\varphi$ at $\zeta$ and denoted by $\varphi^{\prime}(\zeta)$. It satisfies $\varphi^{\prime}(\zeta)=\left|\varphi^{\prime}(\zeta)\right| \bar{\zeta} \eta$ with $\eta=\varphi(\zeta)$. It turns out that the discrete part (that is, mass points, or atoms) of the Aleksandrov-Clark measures associated to $\varphi$ are in correspondence with the finite angular derivatives of $\varphi$.

The map $\varphi$ has a finite angular derivative at $\zeta \in \mathbb{T}$ if and only if there is $\alpha \in \mathbb{T}$ such that $\tau_{\alpha}(\{\zeta\})>0$. In that case $\varphi(\zeta)=\alpha$ and $\left|\varphi^{\prime}(\zeta)\right|=\tau_{\alpha}(\{\zeta\})^{-1}$.

For the proof of this result, convenient references are [3, Theorem 9.2.1; 14, Theorem 3.1], where it is established in conjunction with the Julia-Carathéodory Theorem.

In view of the correspondence between the atoms of the Aleksandrov-Clark measures and angular derivatives, we can characterize the three types of analytic self-maps as follows: $\varphi$ (not the identity) is of

(1) elliptic type if and only if $\tau_{\alpha}(\{\alpha\})<1$ for all $\alpha$;

(2) hyperbolic type if and only if $\tau_{\alpha}(\{\alpha\})>1$ for some (necessarily unique) $\alpha$;

(3) parabolic type if and only if $\tau_{\alpha}(\{\alpha\})=1$ for some (necessarily unique) $\alpha$.

The aim of this work is to examine the dynamical properties of the Aleksandrov-Clark measures associated to self-maps of $\mathbb{D}$ falling into this classification established by the Linear Fractional Model Theorem.

In that sense, we completely determine the convergence, in total variation norm or weak*sense, and after a suitable normalization, of the Aleksandrov-Clark measures belonging to the iterates of $\varphi$. As it will be shown, the associated model will play a prominent role in this analysis. 
Table 1 summarizes our results. By $\tau_{\alpha}^{n}$, we denote the Aleksandrov-Clark measure of the iterate $\varphi_{n}$ at $\alpha \in \mathbb{T}$. In the parabolic case, we use the following terminology, which is explained in more detail in Subsection 5.2: the map $\varphi$ with Denjoy-Wolff point 1 is of finite shift if $\sup _{n}\left\|\tau_{1}^{n}\right\|<\infty$ and of infinite shift otherwise.

\section{Preliminaries}

\subsection{Iteration of Aleksandrov-Clark measures}

In order to understand the behaviour of Aleksandrov-Clark measures under the iteration of the symbol, we will find it useful to interpret these measures from the viewpoint of composition operators. The idea is due to Sarason [15] and goes as follows: Let $\mu \in \mathcal{M}$, the space of all complex Borel measures on $\mathbb{T}$ endowed with the total variation norm. Then the Poisson integral $u(z)=\int_{\mathbb{T}} P_{z} d \mu$ defines a harmonic function on $\mathbb{D}$. If $\varphi: \mathbb{D} \rightarrow \mathbb{D}$ is an analytic map, then the composition $u \circ \varphi$ is also harmonic, and it is easy to see that it can be represented as the Poisson integral of a unique measure $\nu \in \mathcal{M}$. In this setting, Sarason defined $C_{\varphi} \mu=\nu$ and showed that the resulting linear operator $C_{\varphi}$ acts boundedly on $\mathcal{M}$.

To describe the action of $C_{\varphi}$ on the unit circle, note that the correspondence $C_{\varphi} \mu=\nu$ above can be written as

$$
\begin{aligned}
\int_{\mathbb{T}} P_{z} d \nu & =\int_{\mathbb{T}} P_{\varphi(z)} d \mu \\
& =\int_{\mathbb{T}}\left(\int_{\mathbb{T}} P_{z} d \tau_{\alpha}\right) d \mu(\alpha),
\end{aligned}
$$

for all $z \in \mathbb{D}$, where the second equality follows from the definition of the family $\left\{\tau_{\alpha}\right\}$ of Aleksandrov-Clark measures. Approximating any continuous function $f$ on $\mathbb{T}$ by linear combinations of Poisson kernels, we arrive at the identity

$$
\int_{\mathbb{T}} f d \nu=\int_{\mathbb{T}}\left(\int_{\mathbb{T}} f d \tau_{\alpha}\right) d \mu(\alpha)
$$

\begin{tabular}{|c|c|c|}
\hline Model for $\varphi$ & \multicolumn{2}{|c|}{ Convergence of the associated Aleksandrov-Clark measures } \\
\hline & $\varphi$ not inner: & $\varphi$ inner: \\
\hline Elliptic & $\begin{array}{l}\text { For any } \alpha \in \mathbb{T} \text {, } \\
\tau_{\alpha}^{n} \text { is convergent in norm }\end{array}$ & $\begin{array}{l}\text { For any } \alpha \in \mathbb{T}, \\
\tau_{\alpha}^{n} \text { is only weak-convergent }\end{array}$ \\
\hline $\begin{array}{l}\text { Hyperbolic } \\
\text { Denjoy-Wolff Point } 1 \\
\varphi(1)=1, \varphi^{\prime}(1)<1 \\
c=1 / \varphi^{\prime}(1)\end{array}$ & $\begin{array}{l}\text { For any } \alpha \in \mathbb{T} \backslash\{1\}, \tau_{\alpha}^{n} \text { is } \\
\tau_{1}^{n} \text { is NOT convergent } \\
\frac{\tau_{1}^{n}}{c^{n}} \text { is convergent in norm } \\
\frac{\tau_{1}^{n}}{\left\|\tau_{1}^{n}\right\|} \text { is weak }{ }^{*} \text {-convergent }\end{array}$ & $\begin{array}{l}\text { norm to } 0 \\
\qquad \Leftrightarrow \int_{\mathbb{T} \backslash\{1\}} \log |\theta| d \tau_{1}\left(e^{i \theta}\right)>-\infty\end{array}$ \\
\hline $\begin{array}{l}\text { Parabolic } \\
\text { Denjoy-Wolff Point } 1 \\
\varphi(1)=1, \varphi^{\prime}(1)=1\end{array}$ & $\begin{array}{l}\text { Finite shift case: } \\
\text { For any } \alpha \in \mathbb{T}, \\
\tau_{\alpha}^{n} \text { is convergent in norm }\end{array}$ & $\begin{array}{l}\text { Infinite shift case: } \\
\text { For any } \alpha \in \mathbb{T} \backslash\{1\}, \\
\tau_{\alpha}^{n} \text { is convergent in norm to } 0 \\
\tau_{1}^{n} \text { is NOT convergent } \\
\frac{\tau_{1}^{n}}{\left\|\tau_{1}^{n}\right\|} \text { is weak*-convergent }\end{array}$ \\
\hline
\end{tabular}

TABLE 1. Convergence of Aleksandrov-Clark measures 
In fact, an argument based on the monotone class theorem (cf., for example, [3, Section 9.4]) shows that this holds true for every bounded Borel function $f$ on $\mathbb{T}$. Henceforth, we agree to write briefly

$$
C_{\varphi} \mu=\nu=\int_{\mathbb{T}} \tau_{\alpha} d \mu(\alpha) .
$$

As a consequence of (2.1), we have the identity $\tau_{\alpha}=C_{\varphi} \delta_{\alpha}$ as well as the following important recursion formulas.

Lemma 2.1. Let $\varphi$ be an analytic self-map of $\mathbb{D}$. Then, for $n \geqslant 2$, the Aleksandrov-Clark measure associated to $\varphi_{n}$ at $\alpha \in \mathbb{T}$ satisfies $\tau_{\alpha}^{n}=C_{\varphi} \tau_{\alpha}^{n-1}=C_{\varphi_{n-1}} \tau_{\alpha}$, that is,

$$
\tau_{\alpha}^{n}=\int_{\mathbb{T}} \tau_{\xi} d \tau_{\alpha}^{n-1}(\xi)=\int_{\mathbb{T}} \tau_{\xi}^{n-1} d \tau_{\alpha}(\xi)
$$

Corollary 2.2. Suppose that $\tau_{1}(\{1\})=c>0$, or equivalently $\varphi(1)=1$ and $\varphi^{\prime}(1)=1 / c$. Then, for $n \geqslant 2$,

$$
\tau_{1}^{n}=c \tau_{1}^{n-1}+\nu_{n} \quad \text { with } \quad \nu_{n}=\int_{\mathbb{T} \backslash\{1\}} \tau_{\xi}^{n-1} d \tau_{1}(\xi) .
$$

Hence, $\tau_{1}^{n}$ can be expressed as the following sum of positive measures:

$$
\tau_{1}^{n}=c^{n} \delta_{1}+c^{n-1} \nu_{1}+c^{n-2} \nu_{2}+\cdots+\nu_{n},
$$

where $\nu_{1}$ is the restriction of $\tau_{1}$ to $\mathbb{T} \backslash\{1\}$.

REMARK 2.3. It is of interest to note that the singular parts of $\tau_{1}^{n-1}$ and $\nu_{n}$ above have disjoint supports in the sense of measure. Indeed, if

$$
E_{n-1}=\left\{\zeta \in \mathbb{T}: \varphi_{n-1}(\zeta)=1\right\},
$$

then $\left(\tau_{1}^{n-1}\right)^{s}\left(\mathbb{T} \backslash E_{n-1}\right)=0$, while $\left(\tau_{\xi}^{n-1}\right)^{s}\left(E_{n-1}\right)=0$ for each $\xi \neq 1$ and hence $\nu_{n}^{s}\left(E_{n-1}\right)=0$. As a consequence, the measures arising in the above expansion for $\tau_{1}^{n}$ have singular parts with disjoint supports.

\subsection{Half-plane model for hyperbolic and parabolic types}

Assume that $\varphi$ is of either hyperbolic or parabolic type with Denjoy-Wolff point 1 and $\varphi^{\prime}(1)=1 / c \in(0,1]$. This means that $\tau_{1}(\{1\})=c \geqslant 1$ and hence

$$
\frac{1+\varphi(z)}{1-\varphi(z)}=c \frac{1+z}{1-z}+i a+\int_{\mathbb{T} \backslash\{1\}} \frac{\zeta+z}{\zeta-z} d \tau_{1}(\zeta),
$$

where $a=\operatorname{Im}(1+\varphi(0)) /(1-\varphi(0))$.

We often transform this into the right half-plane $\mathbb{H}$ by sending the Denjoy-Wolff point 1 to $\infty$ via the Cayley transformations

$$
w=\frac{1+z}{1-z}, \quad z=\frac{w-1}{w+1}, \quad \Phi(w)=\frac{1+\varphi(z)}{1-\varphi(z)}, \quad \varphi(z)=\frac{\Phi(w)-1}{\Phi(w)+1} .
$$

Then $\Phi$ maps $\mathbb{H}$ into itself and is of the form

$$
\Phi(w)=c w+i a+G(w)=c w+\Gamma(w),
$$

where $G$ and $\Gamma=i a+G$ have the property that

$$
\angle \lim _{w \rightarrow \infty} \frac{G(w)}{w}=\angle \lim _{w \rightarrow \infty} \frac{\Gamma(w)}{w}=0 .
$$

Here $w$ approaches $\infty$ non-tangentially, that is, within a Stolz angle $\{w:|\operatorname{Im} w|<\beta \operatorname{Re} w\}$ for some $\beta>0$. Thus $\Phi$ has $\infty$ as its Denjoy-Wolff point with the angular derivative $\Phi^{\prime}(\infty)=c$. (See $[\mathbf{2}$, Section 4].) 
If necessary, the integral defining $G$ above can be transformed into an integral calculated over the real line. More precisely, writing $w=x+i y$ with $x>0$ we have

$$
G(w)=\int_{\mathbb{R}} \frac{i \eta w-1}{i \eta-w} d \tilde{\tau}_{1}(\eta)=U(w)+i V(w),
$$

where

$$
\begin{aligned}
& U(w)=\int_{\mathbb{R}} \frac{x}{x^{2}+(\eta-y)^{2}}\left(1+\eta^{2}\right) d \tilde{\tau}_{1}(\eta), \\
& V(w)=\int_{\mathbb{R}}\left\{-\eta+\frac{(\eta-y)\left(1+\eta^{2}\right)}{x^{2}+(\eta-y)^{2}}\right\} d \tilde{\tau}_{1}(\eta),
\end{aligned}
$$

and $\tilde{\tau}_{1}$ is the pull-back of $\tau_{1}$ to $\mathbb{R}$, that is,

$$
d \tilde{\tau}_{1}(\eta)=d \tau_{1}\left(\frac{i \eta-1}{i \eta+1}\right)
$$

It should be noted that here $\tilde{\tau}_{1}$ can be any positive Borel measure with finite mass on the real line. The integrals defining $U$ and $V$ make sense because the integrands are bounded functions of $\eta$ for each fixed $w$. However, the integral $\int \eta d \tilde{\tau}_{1}(\eta)$ is not necessarily well defined, and hence a different normalization for $V$ cannot always be used (cf. the discussion in [7, p. 105]).

\section{Warm-up: the elliptic case}

If $\varphi$ is not an automorphism and fixes a point $p \in \mathbb{D}$ (that is, $\varphi$ is of elliptic type), then $\varphi_{n}$ converges to $p$ uniformly on compact sets and therefore for each $\alpha \in \mathbb{T}$,

$$
\lim _{n \rightarrow \infty} \int_{\mathbb{T}} P_{z} d \tau_{\alpha}^{n}=\lim _{n \rightarrow \infty} \frac{1-\left|\varphi_{n}(z)\right|^{2}}{\left|\alpha-\varphi_{n}(z)\right|^{2}}=\frac{1-|p|^{2}}{|\alpha-p|^{2}}
$$

for all $z \in \mathbb{D}$. Hence, the sequence $\left\{\tau_{\alpha}^{n}\right\}$ converges in the weak*-topology to the measure $\left(\left(1-|p|^{2}\right) /|\alpha-p|^{2}\right) m$. One may ask if $\left\{\tau_{\alpha}^{n}\right\}$ converges in the norm of $\mathcal{M}$. Having in mind that the Aleksandrov-Clark measures associated to inner functions are singular, one easily deduces the next result which says that it is not always the case.

Proposition 3.1. Let $\varphi$ be a non-automorphic inner function fixing $p \in \mathbb{D}$. For any $\alpha \in \mathbb{T}$, the sequence of Aleksandrov-Clark measures $\left\{\tau_{\alpha}^{n}\right\}$ associated to $\varphi_{n}$ at $\alpha$ does not converge in norm.

Nevertheless, if $\varphi$ is not inner, then the answer is positive.

Proposition 3.2. Let $\varphi$ be an analytic self-map of $\mathbb{D}$ fixing $p \in \mathbb{D}$. Assume that $\varphi$ is not an inner function. Then, for any $\alpha \in \mathbb{T}$, the sequence of Aleksandrov-Clark measures $\left\{\tau_{\alpha}^{n}\right\}$ associated to $\varphi_{n}$ at $\alpha$ converges in norm to $\left(\left(1-|p|^{2}\right) /|\alpha-p|^{2}\right) m$.

Proof. We first assume that $\varphi(0)=0$ and show that $\left\{\tau_{\alpha}^{n}\right\}$ converges to $m$ in norm. Let $\mathcal{M}_{0}$ denote the subspace of $\mathcal{M}$ consisting of measures $\mu$ such that $\mu(\mathbb{T})=0$; or equivalently $P[\mu](0)=0$ where $P[\mu]$ is the Poisson integral of $\mu$ :

$$
P[\mu](z)=\int_{\mathbb{T}} P_{z}(\zeta) d \mu(\zeta)
$$

Since $\varphi(0)=0, C_{\varphi}$ is taking $\mathcal{M}_{0}$ into itself. Moreover, having in mind that $C_{\varphi_{n}} \delta_{\alpha}=\tau_{\alpha}^{n}$ for every $n \geqslant 1$ as well as trivially $C_{\varphi} m=m$, one deduces that

$$
\left\|\tau_{\alpha}^{n}-m\right\|=\left\|C_{\varphi_{n}}\left(\delta_{\alpha}-m\right)\right\| \leqslant\left\|C_{\varphi} \mid \mathcal{M}_{0}\right\|^{n}\left\|\delta_{\alpha}-m\right\| .
$$


It is enough to check that $\left\|C_{\varphi} \mid \mathcal{M}_{0}\right\|<1$. Since $\varphi$ is non-inner, there exists a set $E \subset \mathbb{T}$ of positive Lebesgue measure such that, for each $\zeta \in E$, one has $|\varphi(\zeta)|<1$ and hence

$$
g(\zeta)=\inf _{\xi \in \mathbb{T}} \tau_{\xi}^{a}(\zeta)=\inf _{\xi \in \mathbb{T}} \frac{1-|\varphi(\zeta)|^{2}}{|\xi-\varphi(\zeta)|^{2}}>0
$$

Put $g=0$ on $\mathbb{T} \backslash E$. Then, for $\mu \in \mathcal{M}_{0}$, we have (in the sense of (2.1))

$$
C_{\varphi} \mu=\int_{\mathbb{T}} \tau_{\xi} d \mu(\xi)=\int_{\mathbb{T}}\left(\tau_{\xi}-g \cdot m\right) d \mu(\xi)
$$

Note that each of the measures $\tau_{\xi}-g \cdot m$ is positive. Hence,

$$
\left\|C_{\varphi} \mid \mathcal{M}_{0}\right\| \leqslant \sup _{\xi \in \mathbb{T}}\left\|\tau_{\xi}-g \cdot m\right\|=1-\int_{\mathbb{T}} g d m<1
$$

because $\left\|\tau_{\xi}\right\|=1$. This completes the proof in the case when $\varphi(0)=0$.

Now assume that $p \neq 0$ and define $\psi=\sigma_{p} \circ \varphi \circ \sigma_{p}$ where $\sigma_{p}$ is the disc automorphism that exchanges $p$ and 0 . Then $\psi(0)=0$ and $\varphi_{n}=\sigma_{p} \circ \psi_{n} \circ \sigma_{p}$ for each $n$. Hence, $\tau_{\alpha}^{n}=C_{\varphi_{n}} \delta_{\alpha}=$ $C_{\sigma_{p}} C_{\psi_{n}} C_{\sigma_{p}} \delta_{\alpha}$. Here it is easy to verify that

$$
C_{\sigma_{p}} \delta_{\alpha}=\frac{1-|p|^{2}}{|\alpha-p|^{2}} \delta_{\sigma_{p}(\alpha)}
$$

This completes the proof since $C_{\sigma_{p}} C_{\psi_{n}} \delta_{\sigma_{p}(\alpha)} \rightarrow C_{\sigma_{p}} m=m$ in norm by the first part of the proof.

\section{The hyperbolic case}

In this section, we will assume that $\varphi$ is an analytic self-map of $\mathbb{D}$ of hyperbolic type, that is, $\varphi$ has no fixed point in $\mathbb{D}$ and the angular derivative at its Denjoy-Wolff point is strictly less than 1. Without loss of generality, we will assume throughout this section that 1 is the Denjoy-Wolff point of $\varphi$.

Our first statement concerns the sequence of Aleksandrov-Clark measures associated to a point $\alpha \in \mathbb{T} \backslash\{1\}$. It is based on the fact that the norm of $\tau_{\alpha}^{n}$ is given by

$$
\left\|\tau_{\alpha}^{n}\right\|=\frac{1-\left|\varphi_{n}(0)\right|^{2}}{\left|\alpha-\varphi_{n}(0)\right|^{2}}
$$

Proposition 4.1. Let $\varphi$ be an analytic self-map of $\mathbb{D}$ with Denjoy-Wolff point 1. For any $\alpha \in \mathbb{T} \backslash\{1\}$, the sequence of Aleksandrov-Clark measures $\left\{\tau_{\alpha}^{n}\right\}$ converges to 0 in norm.

Therefore, in both parabolic and hyperbolic types, we are reduced to study the convergence of the sequence of measures $\left\{\tau_{1}^{n}\right\}$.

\subsection{The model}

If $\varphi$ is of hyperbolic type with Denjoy-Wolff point 1 and $c=1 / \varphi^{\prime}(1)$, then $\tau_{1}(\{1\})=c>1$. The half-plane model for $\varphi$ is of the form

$$
\Phi(w)=c w+\Gamma(w)
$$

where $\Gamma: \mathbb{H} \rightarrow \mathbb{H}$ is analytic with angular derivative 0 at infinity, that is, $\Gamma(w) / w \rightarrow 0$ as $w \rightarrow \infty$ non-tangentially (see Subsection 2.2). Moreover, by Corollary 2.2, the AleksandrovClark measures of the iterates $\varphi_{n}$ at 1 have the expansion

$$
\tau_{1}^{n}=c^{n} \delta_{1}+c^{n-1} \nu_{1}+c^{n-2} \nu_{2}+\cdots+\nu_{n}
$$


Obviously, $\left\|\tau_{1}^{n}\right\| \geqslant c^{n}$ and hence $\left\|\tau_{1}^{n}\right\| \rightarrow \infty$ as $n \rightarrow \infty$. So, in order to discuss any sort of convergence, we have to normalize the sequence $\left\{\tau_{1}^{n}\right\}$. As we will see, the results depend strongly on the normalization method.

\subsection{A function-theoretic normalization}

We first study the normalized sequence of measures

$$
\varphi_{n}^{\prime}(1) \tau_{1}^{n}=\frac{\tau_{1}^{n}}{c^{n}}
$$

for $n \geqslant 1$. This normalization goes back to classical works of Koenigs, Wolff and Valiron, and from a function-theoretic perspective can be considered 'a natural one' since it corresponds to the maps $\varphi_{n} / \varphi_{n}^{\prime}(1)$. However, it does not always work: it is not always the case that

$$
\sup _{n} \frac{\left\|\tau_{1}^{n}\right\|}{c^{n}}=\sup _{n} \frac{\operatorname{Re} \Phi_{n}(1)}{c^{n}}<\infty ;
$$

for instance, see the discussion by Valiron [17] (starting on p. 120), where the mapping

$$
\Phi(w)=2 w+\frac{w}{\log (3+w)}
$$

provides a counter-example to (4.1).

In fact, it was later shown by Pommerenke [13] that (4.1) holds if and only if

$$
\int_{1}^{\infty} \frac{|\Phi(t)-c t|}{t^{2}} d t=\int_{1}^{\infty} \frac{|\Gamma(t)|}{t^{2}} d t<\infty
$$

This can be viewed as a regularity requirement imposed on $\varphi$ at its Denjoy-Wolff point; for instance, it is seen to be fulfilled if $\varphi$ satisfies an angular smoothness condition of order $1+\varepsilon$ at 1 for some $\varepsilon>0$, which in terms of the half-plane model means that $\Gamma(w) /|w|^{1-\varepsilon} \rightarrow 0$ as $w \rightarrow \infty$ non-tangentially (cf., for example, [2, p. 51]).

Next result establishes that the condition (4.1) is equivalent to a sort of regularity of the measure $\tau_{1}$.

Proposition 4.2. Suppose that the Aleksandrov-Clark measure $\tau_{1}$ of $\varphi$ satisfies $\tau_{1}(\{1\})=c>1$. Then $\sup _{n}\left\|\tau_{1}^{n}\right\| / c^{n}<\infty$ if and only if

$$
\int_{\mathbb{T} \backslash\{1\}} \log |\theta| d \tau_{1}\left(e^{i \theta}\right)>-\infty .
$$

Proof. We prove that the proposed condition is equivalent to Pommerenke's condition (4.3). After performing the change of variables $t=(1+r) /(1-r)$, we see that $(4.3)$ is equivalent to the pair of conditions

$$
\int_{0}^{1} \operatorname{Re} \gamma(r) d r<\infty \text { and } \int_{0}^{1}|\operatorname{Im} \gamma(r)| d r<\infty
$$

where (see Subsection 2.2)

$$
\gamma(r)=\int_{\mathbb{T} \backslash\{1\}} \frac{e^{i \theta}+r}{e^{i \theta}-r} d \tau_{1}\left(e^{i \theta}\right)=\int_{\mathbb{T} \backslash\{1\}} P_{r}\left(e^{i \theta}\right) d \tau_{1}\left(e^{i \theta}\right)+i \int_{\mathbb{T} \backslash\{1\}} \frac{2 r \sin \theta}{\left|e^{i \theta}-r\right|^{2}} d \tau_{1}\left(e^{i \theta}\right) .
$$

Note that in the denominator we may estimate $\left|e^{i \theta}-r\right|^{2} \simeq(1-r)^{2}+\theta^{2}$ for $|\theta| \leqslant \pi$ (meaning that the two functions are bounded by a constant multiple of each other). Indeed, one may write $\left|e^{i \theta}-r\right|^{2}=(1-r)^{2}+2 r(1-\cos \theta)$ and proceed by using the fact that $1-\cos \theta \simeq \theta^{2}$.

Consequently, for the Poisson kernel we have the estimate

$$
\int_{0}^{1} P_{r}\left(e^{i \theta}\right) d r \simeq \int_{0}^{1} \frac{(1-r) d r}{(1-r)^{2}+\theta^{2}}=\frac{1}{2} \log \left(1+\theta^{2}\right)-\log |\theta|,
$$


whenever $|\theta| \leqslant \pi$. This, along with an application of Fubini's theorem, shows that the first part of (4.4) is equivalent to the condition of the proposition; observe that the function $\frac{1}{2} \log \left(1+\theta^{2}\right)$ is bounded and hence plays no role in integrability. On the other hand, since

$$
\int_{0}^{1} \frac{|2 r \sin \theta|}{\left|e^{i \theta}-r\right|^{2}} d r \leqslant C \int_{0}^{1} \frac{|\theta| d r}{(1-r)^{2}+\theta^{2}} \leqslant C \int_{0}^{\infty} \frac{d s}{s^{2}+1}<\infty
$$

with $C>0$ being a constant independent of $\theta$ for $|\theta| \leqslant \pi$, we see that the second part of (4.4) is always satisfied. This completes the proof.

Theorem 4.3. Let $\varphi$ be of hyperbolic type with Denjoy-Wolff point 1 and $\varphi^{\prime}(1)=1 / c$. If $\sup _{n}\left\|\tau_{1}^{n}\right\| / c^{n}<\infty$, then the sequence $\left\{\tau_{1}^{n} / c^{n}\right\}$ converges in norm to a positive measure $\mu \in \mathcal{M}$, satisfying

$$
c \mu=\int_{\mathbb{T}} \tau_{\xi} d \mu(\xi)
$$

and $\mu(\{1\})=1$.

Proof. Since the measures $\tau_{1}^{n+1} / c^{n+1}-\tau_{1}^{n} / c^{n}$ are positive for all $n$, the existence of the limit measure follows from the boundedness assumption by the completeness of $\mathcal{M}$. By Lemma 2.1, we have the identity

$$
c \frac{\tau_{1}^{n+1}}{c^{n+1}}=\int_{\mathbb{T}} \tau_{\xi} \frac{d \tau_{1}^{n}(\xi)}{c^{n}},
$$

and so formula (4.5) is obtained by passing to the limit as $n \rightarrow \infty$.

Relation (4.5) gives rise to Schröder's functional equation $c \sigma=\sigma \circ \varphi$ in a straightforward way. Indeed, in terms of composition operators (see Subsection 2.1), (4.5) says that $c \mu=C_{\varphi} \mu$. So, if $\sigma: \mathbb{D} \rightarrow \mathbb{H}$ is defined as the Herglotz integral of $\mu$, that is,

$$
\sigma(z)=\int_{\mathbb{T}} \frac{\zeta+z}{\zeta-z} d \mu(\zeta),
$$

then we must have $c \operatorname{Re} \sigma=\operatorname{Re} \sigma \circ \varphi$. Schröder's equation is then obtained by adding a suitable imaginary constant to $\sigma$.

Next example provides a map $\varphi$ of hyperbolic type whose Aleksandrov-Clark measure fails the logarithmic integrability condition of Proposition 4.2. Such a map can be seen as an alternative to Valiron's example (4.2), showing that 'the natural normalization' $\Phi_{n} / c^{n}$ does not always yield a finite limit as $n \rightarrow \infty$.

EXAMPLE 4.4. We define $\varphi$ in the form

$$
\frac{1+\varphi(z)}{1-\varphi(z)}=2 \frac{1+z}{1-z}+\int_{\mathbb{T}} \frac{\zeta+z}{\zeta-z} d \nu(\zeta),
$$

where $\nu$ is the discrete measure $\nu=\sum_{k=1}^{\infty} k^{-2} \delta_{\zeta_{k}}$ with $\zeta_{k}=\exp \left(2^{-k} i\right)$. Then the AleksandrovClark measure associated to $\varphi$ at 1 is $\tau_{1}=2 \delta_{1}+\nu$. In particular, $\varphi$ is of hyperbolic type with Denjoy-Wolff point 1 and $\varphi^{\prime}(1)=\frac{1}{2}$. A simple computation shows that

$$
\int_{\mathbb{T}} \log |\theta| d \nu\left(e^{i \theta}\right)=\sum_{k}\left(\log 2^{-k}\right) k^{-2}=-\infty .
$$




\subsection{A stronger normalization}

Let again $\varphi$ be of hyperbolic type with Denjoy-Wolff point 1, and consider the probability measures

$$
\mu_{n}=\frac{\tau_{1}^{n}}{\left\|\tau_{1}^{n}\right\|}=\frac{\left|1-\varphi_{n}(0)\right|^{2}}{1-\left|\varphi_{n}(0)\right|^{2}} \tau_{1}^{n}
$$

From a purely measure-theoretic perspective, this might appear to be a more appropriate normalization than the one considered above.

It can be shown that the sequence $\left\{\mu_{n}\right\}$ always converges in weak*-sense to a non-trivial measure $\mu$. Indeed, in terms of the Cayley transforms (2.2) we may write

$$
\int_{\mathbb{T}} P_{z} d \mu_{n}=\frac{\operatorname{Re} \Phi_{n}(w)}{\operatorname{Re} \Phi_{n}(1)}
$$

and it is known that this quotient converges to a finite limit for each $w \in \mathbb{H}$ (see, for example, [12]). Moreover, the intertwining relation (4.5) still holds. This is seen by letting $n \rightarrow \infty$ in the identity

$$
\frac{\operatorname{Re} \Phi_{n+1}(1)}{\operatorname{Re} \Phi_{n}(1)} \mu_{n+1}=\int_{\mathbb{T}} \tau_{\xi} d \mu_{n}(\xi)
$$

and noting that the quotient $\operatorname{Re} \Phi_{n+1}(1) / \operatorname{Re} \Phi_{n}(1)=\operatorname{Re} \Phi\left(\Phi_{n}(1)\right) / \operatorname{Re} \Phi_{n}(1)$ tends to $c$ by the Julia-Carathéodory Theorem since $\Phi_{n}(1)$ is known to converge to $\infty$ non-tangentially (see, for example, [16, Proposition 5.2]).

\section{The parabolic case}

In this section, we will assume that $\varphi$ is an analytic self-map of $\mathbb{D}$ of parabolic type, that is, $\varphi$ has no fixed point in $\mathbb{D}$ and the angular derivative at its Denjoy-Wolff point is 1 . Without loss of generality, we will assume throughout this section that 1 is the Denjoy-Wolff point of $\varphi$.

As we already pointed out in Proposition 4.1, for any $\alpha \in \mathbb{T} \backslash\{1\}$, the sequence of Aleksandrov-Clark measures $\left\{\tau_{\alpha}^{n}\right\}$ converges to zero in the norm of $\mathcal{M}$. Therefore, we will focus on the convergence of $\left\{\tau_{1}^{n}\right\}$.

\subsection{The model}

If $\varphi$ is of parabolic type with Denjoy-Wolff point 1 , then $\tau_{1}(\{1\})=1$. As in the hyperbolic case (see Subsection 4.1), the half-plane model of $\varphi$ is of the form

$$
\Phi(w)=w+\Gamma(w),
$$

where $\Gamma(w) / w \rightarrow 0$ as $w \rightarrow \infty$ non-tangentially, and the Aleksandrov-Clark measure of $\varphi_{n}$ at 1 has the expression given by Corollary 2.2:

$$
\tau_{1}^{n}=\delta_{1}+\nu_{1}+\nu_{2}+\cdots+\nu_{n}
$$

\subsection{Finite vs. infinite shift}

From a measure-theoretic point of view, two natural cases now arise in a way which is similar to the hyperbolic setting in the previous section. Recalling that

$$
\left\|\tau_{1}^{n}\right\|=\operatorname{Re} \Phi_{n}(1)=\frac{1-\left|\varphi_{n}(0)\right|^{2}}{\left|1-\varphi_{n}(0)\right|^{2}}
$$

we may say (as in [4]) that the map $\varphi$ (or $\Phi)$ has

(1) finite shift if

$$
\sup _{n}\left\|\tau_{1}^{n}\right\|=\sup _{n} \operatorname{Re} \Phi_{n}(1)<\infty
$$

(2) infinite shift otherwise. 
We remark that the value $\Phi_{n}(1)$ here could be replaced by $\Phi_{n}(w)$ for any $w \in \mathbb{H}$ (see $[\mathbf{4}$, Proposition 3.2]).

Next result follows similarly to Theorem 4.3 , just taking $c=1$.

Theorem 5.1. Let $\varphi$ be of parabolic type with Denjoy-Wolff point 1. If $\varphi$ has finite shift, then the sequence $\left\{\tau_{1}^{n}\right\}$ converges in norm to a positive measure $\mu \in \mathcal{M}$, satisfying

$$
\mu=\int_{\mathbb{T}} \tau_{\xi} d \mu(\xi)
$$

and $\mu(\{1\})=1$.

We observe that if $\sigma: \mathbb{D} \rightarrow \mathbb{H}$ is the Herglotz integral of $\mu$ above (cf. equation (4.6)), then relation (5.1) yields the functional equation $\sigma+i a=\sigma \circ \varphi$. Moreover, the convergence $\tau_{1}^{n} \rightarrow \mu$ implies that the associated Herglotz integrals converge uniformly on compact sets:

$$
\frac{1+\varphi_{n}(z)}{1-\varphi_{n}(z)}-y_{n}=\Phi_{n}\left(\frac{1+z}{1-z}\right)-y_{n} \longrightarrow \sigma(z),
$$

where $y_{n}=\operatorname{Im}\left(1+\varphi_{n}(0)\right) /\left(1-\varphi_{n}(0)\right)=\operatorname{Im} \Phi_{n}(1)$. It is worth noting that the intertwining map $\sigma$ thus obtained is non-constant; in fact, since $\mu(\{1\})=1$, the map $(\sigma-1) /(\sigma+1)$, which takes $\mathbb{D}$ into itself, has angular derivative 1 at 1 .

The case of infinite shift is much more delicate. Recall from Section 1 that the parabolic self-maps of the disc in general fall into two fundamental subtypes, those with positive or zero hyperbolic step, according to whether the orbits of the map are separated or not relative to the pseudo-hyperbolic metric. Thus, combining the concepts of positive/zero hyperbolic type and finite/infinite shift, there is in principle a classification of parabolic maps into four different classes. Nevertheless, as observed by Poggi-Corradini [10, Proposition 4.1] (see also [4, Proposition 3.3]), maps with finite shift always have positive hyperbolic step.

Instead of the normalization in (5.2), Pommerenke [12] used the normalization

$$
\sigma_{n}(z)=\frac{1}{x_{n}}\left[\Phi_{n}\left(\frac{1+z}{1-z}\right)-y_{n}\right]
$$

where $x_{n}+i y_{n}=\Phi_{n}(1)$. He showed that then the sequence $\left\{\sigma_{n}\right\}$ always converges to an analytic map $\sigma: \mathbb{D} \rightarrow \mathbb{H}$ uniformly on compact sets. In addition:

(1) in the positive hyperbolic step case $\sigma \circ \varphi=\sigma+i a$ for some real $a \neq 0$ (whence $\sigma$ is non-constant);

(2) in the zero hyperbolic step case $\sigma \equiv 1$.

Since $x_{n}=\left\|\tau_{1}^{n}\right\|$, we deduce the following result.

Proposition 5.2. Let $\varphi$ be of parabolic type with Denjoy-Wolff point 1. Then the sequence of measures $\left\{\tau_{1}^{n} /\left\|\tau_{1}^{n}\right\|\right\}$ is weak*-convergent to a positive measure $\mu \in \mathcal{M}$ which satisfies (5.1).

REMARK 5.3. In the case of zero hyperbolic step, the measure $\mu$ above is just the Lebesgue measure $m$ because $\sigma \equiv 1$. To obtain a non-trivial intertwining map, a somewhat trickier normalization can be used, as discovered by Baker and Pommerenke [1]. However, it seems not so straightforward to interpret it from the viewpoint of Aleksandrov-Clark measures.

We also point out that the properties of positive/zero hyperbolic type and finite/infinite shift are not directly determined by regularity properties of the map $\varphi$ (as in the hyperbolic case). However, under certain smoothness assumptions placed on $\varphi$ at its Denjoy-Wolff point, finite shift becomes equivalent to positive hyperbolic step and distinguishing between the two remaining classes becomes easier. See $[\mathbf{2}, \mathbf{4}]$ for results in this direction. 
We end this section with a couple of examples.

ExAmple 5.4. Let $\tau_{1}=\delta_{1}+a \delta_{\alpha}$ with $\alpha \in \mathbb{T} \backslash\{1\}$ and $a>0$, and let $b \in \mathbb{R}$. Define $\varphi: \mathbb{D} \rightarrow$ $\mathbb{D}$ by the formula

$$
\frac{1+\varphi(z)}{1-\varphi(z)}=\frac{1+z}{1-z}+a \frac{\alpha+z}{\alpha-z}+i b
$$

so that $\tau_{1}$ is the Aleksandrov-Clark measure of $\varphi$ at 1. Equivalently, in the right half-plane

$$
\Phi(w)=w+a \frac{i \eta w-1}{i \eta-w}+i b=w+a \frac{1+\eta^{2}}{w-i \eta}+i(b-a \eta),
$$

where $i \eta=(1+\alpha) /(1-\alpha)$ is a point of the imaginary axis. Therefore, $\varphi^{\prime \prime}(1)=i(b-a \eta)$, and $[2$, Theorem 4.4] shows that $\varphi$ has positive hyperbolic step if and only if $b \neq a \eta$. In this case, $\varphi$ is also of finite shift. Note that the hyperbolic step and shift of $\varphi$ depend on all the parameters $a, \alpha$ (or $\eta$ ) and $b$. In the symmetric case $\alpha=-1$ ( or $\eta=0$ ), however, the value of $a$ has no effect.

REMARK 5.5. In the case of zero hyperbolic step, the preceding example shows that the convergence given by Proposition 5.2 cannot be in norm in general. Indeed, all the measures $\tau_{1}^{n}$ there are discrete, though the limiting measure is the Lebesgue measure, as observed in Remark 5.3.

Proposition 5.6. Let $\varphi$ be of parabolic type with Denjoy-Wolff point 1 ; that is, $\tau_{1}(\{1\})=$ 1. Suppose that the absolutely continuous part of $\tau_{1}$ satisfies $d \tau_{1}^{a}=g d m$, where $0 \leqslant g \in L^{1}(\mathbb{T})$ and $g$ is continuous at 1 with $g(1)>0$. Then $\left\|\tau_{1}^{n}\right\| \rightarrow \infty$ as $n \rightarrow \infty$, so $\varphi$ is of infinite shift.

Proof. Recall that $\tau_{1}^{n}=\delta_{1}+\nu_{1}+\nu_{2}+\cdots+\nu_{n}$ with

$$
\nu_{n}=\int_{\mathbb{T} \backslash\{1\}} \tau_{\xi}^{n-1} d \tau_{1}(\xi) .
$$

Now we have

$$
\nu_{n}(\mathbb{T})=\int_{\mathbb{T} \backslash\{1\}} \tau_{\xi}^{n-1}(\mathbb{T}) d \tau_{1}(\xi) \geqslant \int_{\mathbb{T}} \frac{1-\left|\varphi_{n-1}(0)\right|^{2}}{\left|\xi-\varphi_{n-1}(0)\right|^{2}} g(\xi) d m(\xi) \longrightarrow g(1)>0
$$

since $\varphi_{n-1}(0) \rightarrow 1$. Therefore, $\left\|\tau_{1}^{n}\right\|=\tau_{1}^{n}(\mathbb{T}) \rightarrow \infty$.

Acknowledgements. This work was initiated during a post-doctoral research stay of the second author in Universidad de Zaragoza. We thank the Departamento de Matemáticas, Universidad de Zaragoza, for the hospitality shown at that time and the IUMA for the support received. We also thank the Departamento de Análisis Matemático, Universidad Complutense de Madrid, for the hospitality during his visits since 2011.

\section{References}

1. I. N. Baker and Ch. Pommerenke, 'On the iteration of the analytic functions in a halfplane II', J. London Math. Soc. (2) 20 (1979) 255-258.

2. P. S. Bourdon and J. H. Shapiro, 'Cyclic phenomena for composition operators', Mem. Amer. Math. Soc. 596 (1997)

3. J. A. Cima, A. L. Matheson and W. T. Ross, The Cauchy transform (Amer. Math. Soc., Providence, 2006).

4. M. D. Contreras, S. Díaz-Madrigal and Ch. Pommerenke, 'Second angular derivatives and parabolic iteration in the unit disk', Trans. Amer. Math. Soc. 362 (2010) 357-388.

5. C. C. Cowen, 'Iteration and solution of functional equations for functions analytic in the unit disk', Trans. Amer. Math. Soc. 265 (1981) 69-95. 
6. C. C. Cowen and B. D. MacCluer, Composition operators on spaces of analytic functions (CRC Press, 1995).

7. J. B. Garnett, Bounded analytic functions (Academic Press, New York, 1981); revised edition by Springer, New York, 2007.

8. G. Koenigs, 'Recherches sur les intégrales de certaines équationes functionelles', Annale Ecole Normale Superior (Supplément) 3 (1884) 3-41.

9. A. Matheson and M. Stessin, 'Applications of spectral measures', Recent Advances in Operator-Related Function Theory, Contemp. Math. 393 (2006) 15-27.

10. P. Poggi-Corradini, 'Pointwise convergence on the boundary in the Denjoy-Wolff Theorem', Rocky Mountain J. Math. 40 (2010) 1275-1288.

11. A. Poltoratski and D. Sarason, 'Aleksandrov-Clark measures', Recent Advances in Operator-Related Function Theory, Contemp. Math. 393 (2006) 1-14.

12. Ch. Pommerenke, 'On the iteration of analytic functions in a halfplane I', J. London Math. Soc. (2) 19 (1979) 439-447.

13. Сh. Pommerenke, 'On asymptotic iteration of analytic functions in the disk', Analysis 1 (1981) 45-61.

14. E. SAKsman, 'An elementary introduction to Clark measures', Topics in complex analysis and operator theory, Univ. Málaga (2007) 85-136.

15. D. SARASOn, 'Composition operators as integral operators', Analysis and partial differential equations, Lecture Notes in Pure and Appl. Math. 122 (Dekker, New York, 1990) 545-565.

16. J. H. Shapiro, Composition operators and classical function theory (Springer, New York, 1993).

17. G. Valiron, 'Sur l'iteration des fonctions holomorphes dans un demiplan', Bull. Sci. Math. (2) 55 (1931) 105-128.

Eva A. Gallardo-Gutiérrez

Dpto. de Análisis Matemático

Facultad de Matemáticas

Universidad Complutense de Madrid e ICMAT

Plaza de Ciencias 3

28040 Madrid

Spain

eva.gallardo@mat.ucm.es
Pekka J. Nieminen

Department of Mathematics and Statistics

University of Helsinki

Box 68

FI-00014 Helsinki

Finland

pjniemin@cc.helsinki.fi 